\title{
Compact Microstrip Triple-Mode Bandpass Filters Using Dual-Stub-Loaded Spiral Resonators
}

\author{
Kai-da XU ${ }^{1,2}$, Meng-ze $L I^{1}$, Yan-hui LIU ${ }^{* 1}$, Jing $A I^{3}$, Ye-cheng BAI ${ }^{1}$ \\ ${ }^{1}$ Inst. of Electromagnetics and Acoustics \& Dept. of Electronic Science, Xiamen University, Xiamen 361005, China \\ ${ }^{2}$ Shenzhen Research Inst. of Xiamen University, Shenzhen 518057, China \\ ${ }^{3}$ EHF Key Lab of Science, University of Electronic Science and Technology of China, Chengdu 611731, China \\ yanhuiliu@xmu.edu.cn
}

Submitted June 14, 2016 / Accepted December 16, 2016

\begin{abstract}
Two new microstrip triple-mode resonators loaded with T-shaped open stubs using axially and centrally symmetric spiral structures, respectively, are presented. Spiraled for circuit size reduction, these two halfwavelength resonators can both generate three resonant modes over a wide frequency band by loading two T-stubs with different lengths. Due to the structural symmetry, they can be analyzed by odd-and even-mode method. To validate the design concept, two compact bandpass filters (BPFs) using these two novel resonators with center frequencies of $1.76 \mathrm{GHz}$ and $2.44 \mathrm{GHz}$ for the GSM1800 and WLAN/Zigbee applications, respectively, have been designed, fabricated and tested. The center frequencies and bandwidths can be tunable through the analysis of resonant frequency responses, fractional bandwidths and external quality factor versus the resonator parameters. The final measured results have achieved good consistence with the simulations of these two BPFs.
\end{abstract}

\section{Keywords}

Spiral resonator, stub-loaded resonators, transmission zeros, triple-mode bandpass filter

\section{Introduction}

Bandpass filters (BPFs) are basic building blocks in the RF front-end and microwave wireless communication systems. Over the past decade, the BPFs with compact size [1], [2], sharp frequency selectivity [3], [4], low insertion loss and wide bandwidth have been studied and exploited extensively, and various design approaches have been proposed [5-12]. An effective way for compact filter design is that modifying the traditional resonator to generate additional resonant modes, resulting in one resonator with more fundamental resonant frequencies. Dual-mode resonators and filters are the main research topics in recent years, which have been analyzed deeply and comprehensively in numerous reports with a variety of structures, including rectangle loop resonators [5], meander loops [6], triangular loop structures [7], and stub-loaded resonators [8]. Planar microstrip triple-mode or multi-mode filters have encountered many difficulties in the integrated process as they tend to be large in volume and are not easy for integration.

A harmonic-suppressed BPF based on a triple-mode stub-loaded resonator is proposed in [9], which has the advantage that the even-mode frequencies can be flexibly controlled whereas the odd-mode frequencies are fixed. However, it involves complex structure in the design process. In [10], a triple-mode BPF using a modified circular patch resonator is introduced, but the bad rejection in the stopband occurs. Reference [11] presents a wideband BPF with controllable bandwidth and suppression of the harmonic band, in which four same open loop resonators are adopted. However, the frequency selectivity still needs to be improved. In [12], a novel defected open-loop resonator as the slotline configuration is applied to design a compact triple-mode defected ground waveguide resonator-based $\mathrm{BPF}$, which is compact and easy for integration with planar technology. Moreover, a novel triple-mode hexagonal BPF with capacitive loading stubs is introduced in [13], which is developed from a conventional hexagonal loop dual-mode resonator. In [14], a triple mode filter using a spiral resonator loaded with two short-stubs and an open-stub is presented.

In this paper, two different types of modified triplemode spiral resonators have been proposed. The theoretical odd- and even-mode analysis is given to verify the performance of the resonators. Two BPFs using axially and centrally symmetric structures with center frequencies of $1.76 \mathrm{GHz}$ and $2.44 \mathrm{GHz}$ for the GSM1800 and WLAN/Zigbee applications, respectively, have been simulated, fabricated and tested. Good agreements are shown between simulated and experimental results.

\section{Resonators Analysis}

The schematic layouts of two proposed dual-T-stubloaded spiral resonators are shown in Fig. 1, which share the same equivalent structure. Because of the spiral struc- 


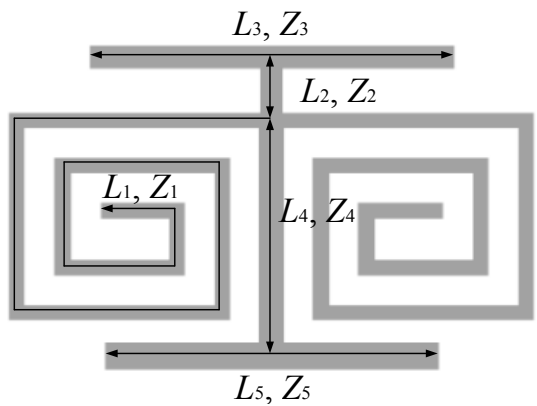

(a)

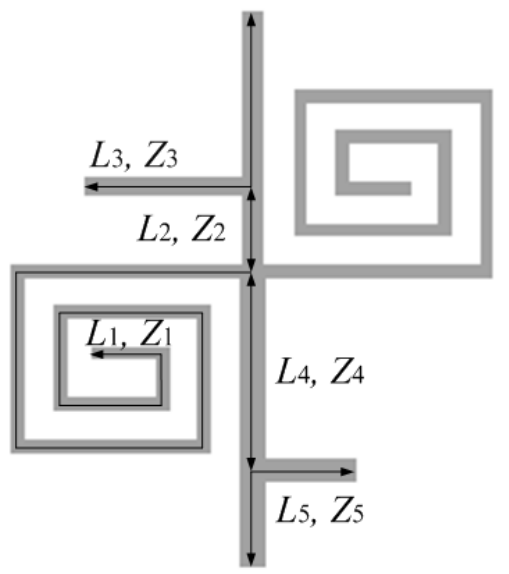

(b)

Fig. 1. Layouts of two proposed spiral resonators: (a) Axially symmetric spiral resonator. (b) Centrally symmetric spiral resonator.

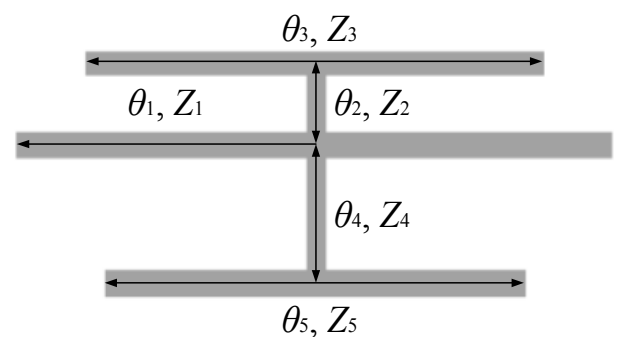

(a)

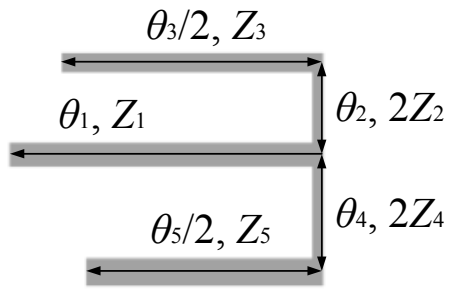

(b)

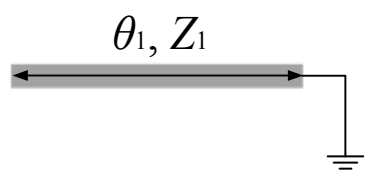

(c)

Fig. 2. Equivalent configuration and equivalent circuits of the proposed spiral resonators: (a) Equivalent configuration. (b) Even-mode equivalent circuit. (c) Odd-mode equivalent circuit. tures, when the electrical lengths are fixed, the sizes of these two proposed resonators can be reduced greatly.

Figure 2(a) illustrates the equivalent configuration of the proposed spiral resonators, which is composed of a uniform microstrip half-wavelength resonator and a pair of T-shaped stubs with different lengths. $\theta_{i}$ refers to the electrical length of $L_{i}$, and $Z_{i}$ denotes the characteristic impedance of the corresponding strips $(i=1,2,3,4$ and 5).

As the equivalent configuration is symmetrical in structure, apparently, we could utilize the odd- and evenmode method to further analyze this configuration. For the even-mode excitation, an approximately equivalent circuit is depicted in Fig. 2(b). We simplify the analysis by setting $Z_{1}=Z_{3}=2 Z_{2}=2 Z_{4}=Z_{5}$, therefore, the input impedances of the two even-mode equivalent circuits $Z_{\text {in_even_1 }}$ and $Z_{\text {in_even_2 }}$ can be deduced as follows:

$$
\begin{aligned}
& Z_{\text {in_even_1 }}=\frac{Z_{1}}{\mathrm{j} \tan \left(\theta_{1}+\theta_{2}+\theta_{3} / 2\right)}, \\
& Z_{\text {in_even_2 }}=\frac{Z_{1}}{\mathrm{j} \tan \left(\theta_{1}+\theta_{4}+\theta_{5} / 2\right)} .
\end{aligned}
$$

Because of the resonance conditions that $\operatorname{Im}\left(Z_{\text {in_even_1 }}\right)=\infty$ and $\operatorname{Im}\left(Z_{\text {in_even_2 }}\right)=\infty$, we can derive the following results:

$$
\begin{aligned}
& f_{\text {even_1 }}=\frac{n c}{2\left(L_{1}+L_{2}+L_{3} / 2\right) \sqrt{\varepsilon_{e f f}}}, \\
& f_{\text {even_2 }}=\frac{n c}{2\left(L_{1}+L_{4}+L_{5} / 2\right) \sqrt{\varepsilon_{\text {eff }}}}
\end{aligned}
$$

where $n=1,2,3 \ldots, c$ is the velocity of light in free space, and $\varepsilon_{\text {eff }}$ denotes the effective dielectric constant of the substrate.

For the odd-mode excitation, its equivalent circuit is depicted in Fig. 2(c). The input impedance of the odd-mode circuit $Z_{\text {in odd }}$ can be obtained as follows:

$$
Z_{\text {in_odd }}=\mathrm{j} Z_{1} \tan \theta_{1}
$$

The resonance condition is $\operatorname{Im}\left(Z_{\text {in_odd }}\right)=\infty$. Therefore, when the odd mode is excited, the resonant frequency can be deduced as:

$$
f_{\text {odd }}=\frac{(2 n-1) c}{4 L_{1} \sqrt{\varepsilon_{e f f}}} .
$$

When $L_{2}+L_{3} / 2<L_{1}<L_{4}+L_{5} / 2$, it can be derived that $f_{\text {even } 2}<f_{\text {odd }}<f_{\text {even } 1}$. Following this analysis, a triple-mode resonator is presented. The above analysis shows that the resonant frequencies of two proposed triple-mode resonators could be changed by tuning the lengths of $L_{1}, L_{2}, L_{3}, L_{4}$, and $L_{5}$.

Figure 3 presents the variations of these three resonant frequencies with respect to $L_{3}$ and $L_{5}$ for the proposed resonators. In Fig. 3(a), by changing the length of $L_{3}$ from 16.8 to $19.2 \mathrm{~mm}, f_{\text {odd }}$ and $f_{\text {even } 2}$ are rarely changed, 
but $f_{\text {even } 1}$ will reduce considerably. Figure $3(\mathrm{~b})$ plots the trend of these three resonant modes likewise. When $L_{5}$ increases from 13.4 to $15.8 \mathrm{~mm}, f_{\text {odd }}$ and $f_{\text {even_1 }}$ will almost keep unchanged, but $f_{\text {even } 2}$ will decline significantly. These simulated results agree well with the deduced equations (3), (4) and (6). Therefore, to design a desired passband of the filter using the proposed structure, we must tailor these three resonant modes initially, which are mainly dominated by the electrical lengths of the resonator and two T-stubs.

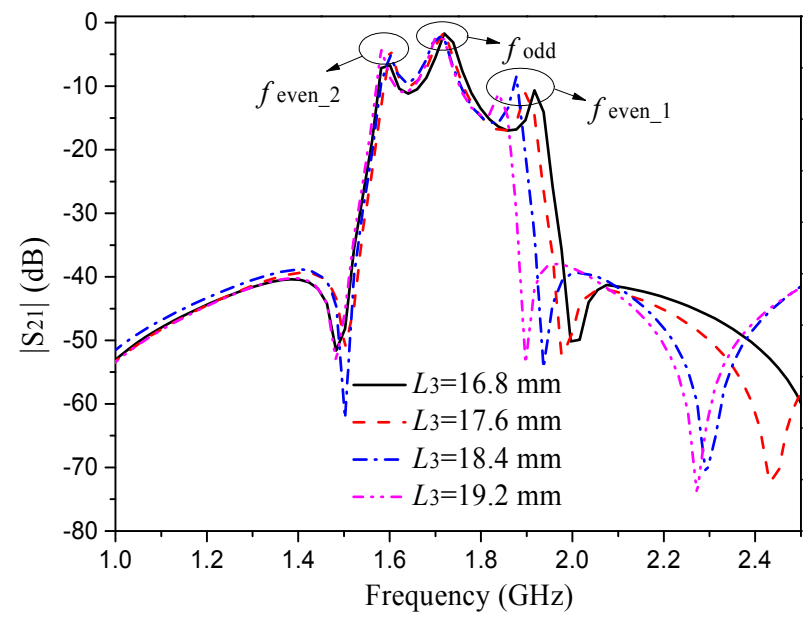

(a)

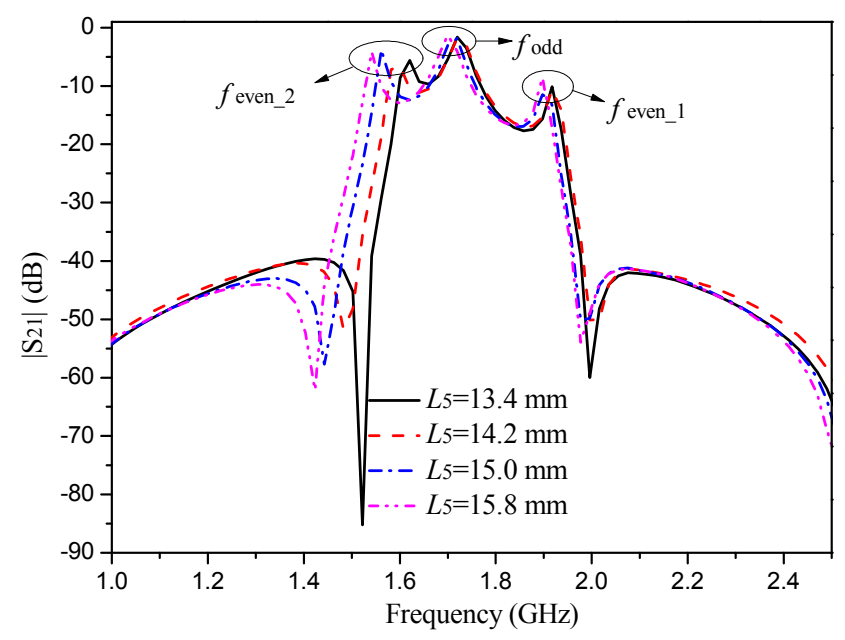

(b)

Fig. 3. Simulated frequency responses of the two proposed triple-mode resonators.

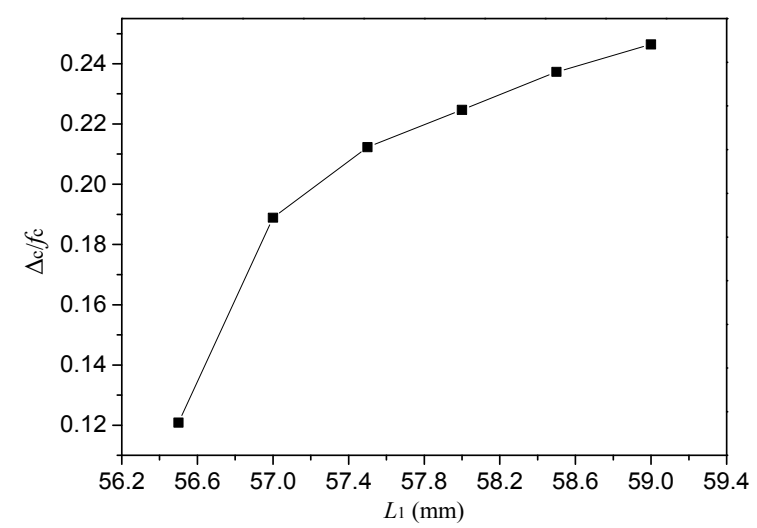

(a)

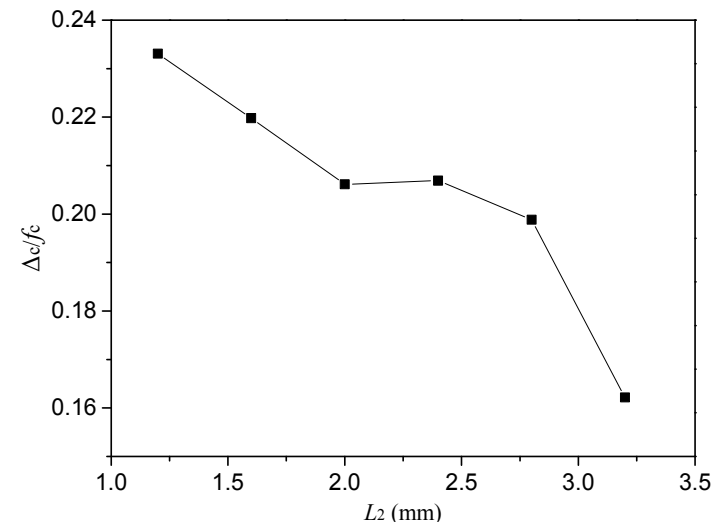

(b)

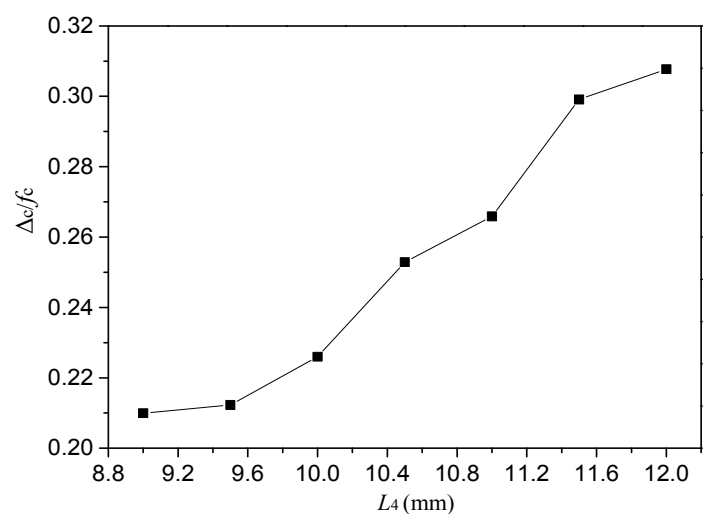

(c)

Fig. 4. Variation of fractional bandwidth $\Delta c / f_{\mathrm{c}}$ with different values of (a) $L_{1}$, (b) $L_{2}$, (c) $L_{4}$

The fractional bandwidth is a key and important technical index in the filter design. If the BPF operates at center frequency $f_{\mathrm{c}}$ between lower cut-off frequency $f_{1}$ and upper cut-off frequency $f_{2}$, where $f_{\mathrm{c}}=\left(f_{1}+f_{2}\right) / 2$, the fractional bandwidth is equal to $\left(f_{2}-f_{1}\right) / f_{\mathrm{c}}$. When we apply the abovementioned resonator structure in filter design, the fractional bandwidth $\Delta c / f_{\mathrm{c}}$ with different varied parameters of the filter has been demonstrated in Fig. 4, where $\Delta c$ is the 3-dB bandwidth, i.e., $\Delta c=f_{2}-f_{1}$. As the length of $L_{1}$ increases from 56.5 to $59.0 \mathrm{~mm}$, the fractional bandwidths will rise up from $12 \%$ to $25 \%$ as seen in Fig. 4(a). In addition, apparently that the fractional bandwidth will decrease from $23.3 \%$ to $16 \%$ when $L_{2}$ increases from 1.2 to $3.2 \mathrm{~mm}$ as shown in Fig. 4(b). For $L_{4}$ in Fig. 4(c), when it changes from 9.0 to $12.0 \mathrm{~mm}$, the fractional bandwidth will also extend to $31 \%$ from original $21 \%$. Therefore, the fractional bandwidth can be tuned flexibly by slightly adjusting the lengths of $L_{1}, L_{2}$, or $L_{4}$.

\section{Two Triple-Mode BPFs Design}

\subsection{BPF using Axially Symmetric Spiral Resonator}

Based on the proposed axially symmetric dual-T-stubloaded spiral resonator, a compact wideband triple-mode bandpass filter is designed on the substrate with a relative 


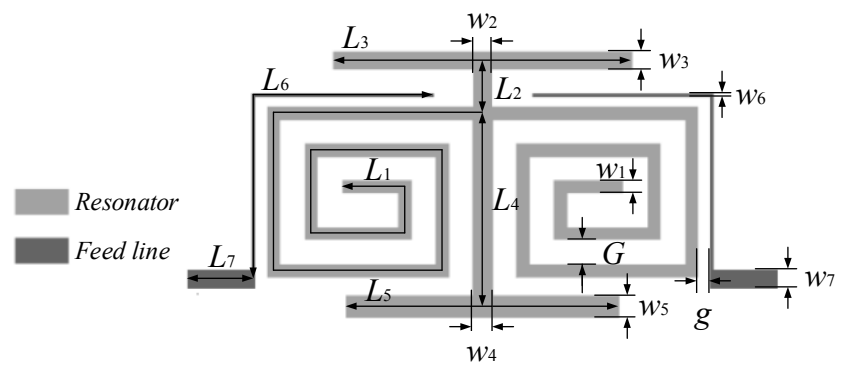

Fig. 5. Layout of the proposed axial symmetric BPF.

dielectric constant of 9.2 and a thickness of $1 \mathrm{~mm}$. The layout of this filter and its dimension parameters are illustrated in Fig. 5. We also analyze the influence of the spiral gap parameter $G$ on the resonance characteristics of the resonator. As depicted in Fig. 6, the upper even mode $f_{\text {even_1 }}$ will almost keep fixed as the value of the gap $G$ changes but the other dimensions remain unchanged. As the parameter $G$ decreases from 1.5 to $0.1 \mathrm{~mm}$, the odd-mode frequency $f_{\text {odd }}$ will decline fast from 1.72 to $1.42 \mathrm{GHz}$, while the lower even-mode frequency $f_{\text {even } 2}$ will decrease from 1.6 to $1.42 \mathrm{GHz}$ slowly. Eventually, these two modes overlap each other at $1.42 \mathrm{GHz}$. Thus, the gap of the spiral resonator can be used to slightly tune the distances among these three resonant frequencies, which can control the bandwidth and center frequency of the proposed filter.

In addition, the bandwidth of this filter can be affected with the coupling gap $g$, and Figure 7 illustrates the extracted external quality factor $Q_{\mathrm{e}}$ with varied $g$. When $g$ increases from 0.1 to $1.2 \mathrm{~mm}$, higher $Q_{\mathrm{e}}$ of the filter will be obtained, which causes the decrease of the bandwidth. Therefore, for filter design, we can obtain the center frequency by tuning the gap $G$ first, and then adjust the bandwidth by changing the coupling gap $g$. After optimization, the parameters of this BPF are finally chosen as follows: $L_{1}=57.5, \quad L_{2}=2.85, \quad L_{3}=16.8, \quad L_{4}=10.45, \quad L_{5}=14.2$, $L_{6}=19.7, L_{7}=3.5, W_{1}=0.7, \quad W_{2}=1, \quad W_{3}=1, \quad W_{4}=1.1$, $W_{5}=1.2, W_{6}=0.2, W_{7}=1, G=1.3, g=0.1$, where the units are all in millimeter.

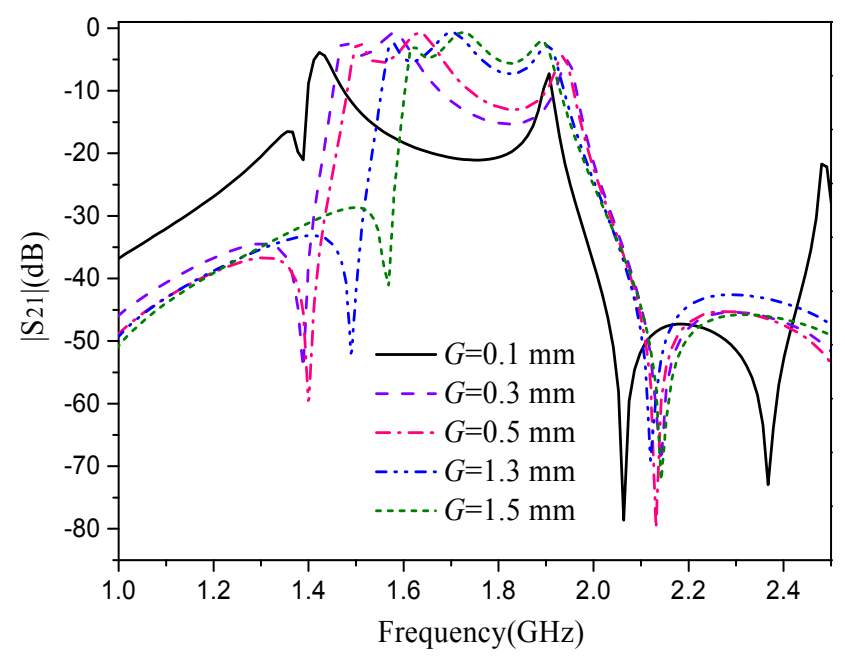

Fig. 6. Simulated frequency responses of the BPF under weak coupling with different $G$.

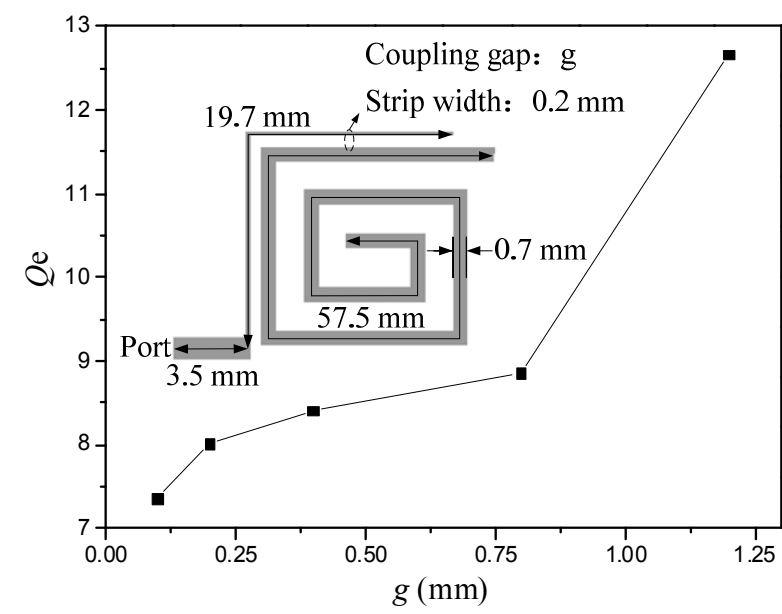

Fig. 7. Extracted external quality factor $Q_{\mathrm{e}}$ with varied $g$.

Figure 8 shows the measured and simulated results of the fabricated axially symmetric BPF. The size of this filter is about $13.9 \mathrm{~mm} \times 23.6 \mathrm{~mm}$ corresponding to a size of $0.2 \lambda_{\mathrm{g}} \times 0.33 \lambda_{\mathrm{g}}$, where $\lambda_{\mathrm{g}}$ is the guided wavelength on the substrate at the center frequency. Seen from the simulated results, we will find that this filter has the center frequency of $1.79 \mathrm{GHz}$ and $3-\mathrm{dB}$ fractional bandwidth of about $22.5 \%(1.59-1.98 \mathrm{GHz})$. Its insertion losses and return losses are less than $0.31 \mathrm{~dB}$ and over $19.3 \mathrm{~dB}$ in the passband, respectively. While the center frequency of the measured results is at $1.76 \mathrm{GHz}$ and the $3-\mathrm{dB}$ bandwidth is around from 1.59 to $1.92 \mathrm{GHz}$. The measured insertion losses and return losses are less than $1.5 \mathrm{~dB}$ and better than $19 \mathrm{~dB}$ in the passband, respectively. Four TZs are introduced due to this unique schematic structure, which are located at 1.03, 1.48, 2.04 and $2.61 \mathrm{GHz}$, respectively. Slight deviation between measurements and simulations could be attributed to the fabrication and measurement error. Figure 9 presents a photograph of the fabricated BPF using axially symmetric spiral resonator. Table 1 tabulates the performance comparisons among the proposed axially symmetric filter (i.e. Filter A) and several other reported triple-mode BPFs.

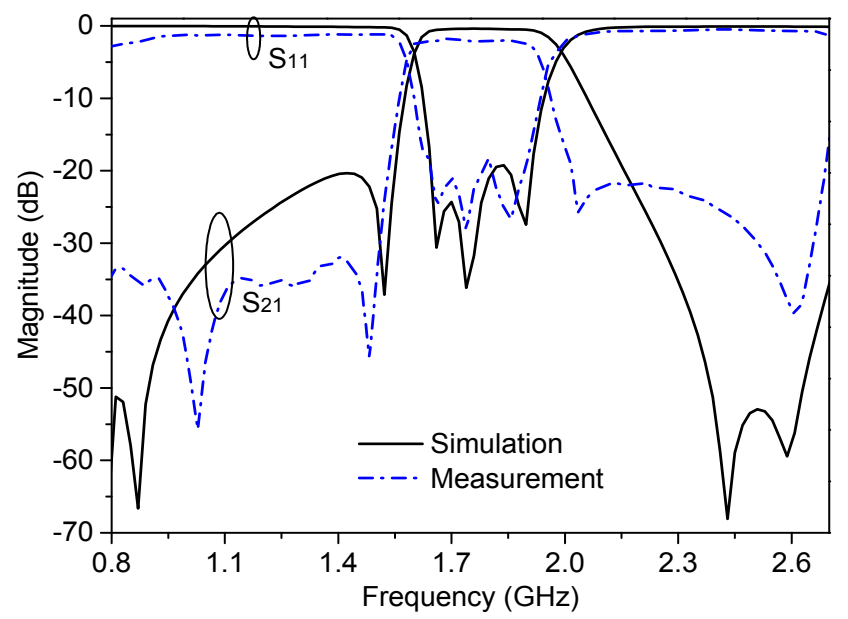

Fig. 8. Measured and simulated results of the proposed filter. 


\begin{tabular}{|c|c|c|c|c|c|c|c|}
\hline Ref. & $\begin{array}{c}\text { Substrate Height } \\
(\mathbf{m m}) / \boldsymbol{\varepsilon}_{\mathbf{r}}\end{array}$ & $\begin{array}{c}\text { Center } \\
\text { Frequency }(\mathbf{G H z})\end{array}$ & $\begin{array}{c}\text { Insertion Loss } \\
(\mathbf{d B})\end{array}$ & Return Loss $\mathbf{( d B )}$ & 3-dB FBW (\%) & $\begin{array}{c}\text { Circuit Size } \\
\left(\lambda_{\mathrm{g}} \times \lambda_{\mathrm{g}}\right)\end{array}$ & $\begin{array}{c}\text { Triple-mode Production } \\
\text { Mechanism }\end{array}$ \\
\hline$[10]$ & $0.625 / 10.2$ & 2.4 & 0.5 & 16 & 29 & $0.34 \times 0.34$ & $\mathrm{DMS}^{1}$ \\
\hline$[11]$ & $1.14 / 3.2$ & 4.17 & 0.6 & 9 & 26 & $0.24 \times 0.61$ & $(2 \mathrm{OLRs}+2 S t u b s)^{2}$ \\
\hline$[12]$ & $0.8 / 4.5$ & 3.5 & 1.9 & 11.5 & 16 & $0.25 \times 0.39$ & $\mathrm{DGW}^{3}$ \\
\hline$[13]$ & $1 / 9.2$ & 2.4 & 2.4 & 17 & 4.8 & $0.29 \times 0.39$ & SPoRR $^{4}$ \\
\hline Filter A & $1 / 9.2$ & 1.76 & 1.5 & 19 & 18.8 & $0.2 \times 0.33$ & $\mathrm{DSLSR}^{5}$ \\
\hline
\end{tabular}

${ }^{1}$ DMS: degenerate modes split; ${ }^{2}\left(2 \mathrm{OLRs}+\mathbf{2 S t u b s ) : ~ t w o ~ o p e n ~ l o o p ~ r e s o n a t o r s ~ a n d ~ t w o ~ s t u b s ; ~}{ }^{3} \mathbf{D G W}\right.$ : defected ground waveguide structure; ${ }^{4}$ SPoRR: small perturbation on the ring resonator; ${ }^{5}$ DSLSR: dual-stub-loaded spiral resonator.

Tab. 1. Performance comparisons of some reported triple-mode BPFs.

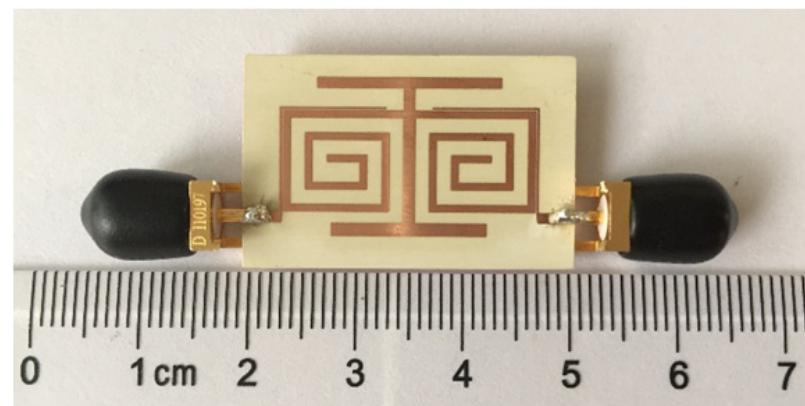

Fig. 9. Photograph of the proposed axially symmetric BPF.

\subsection{BPF using Centrally Symmetric Spiral Resonator}

In this sub-section, another compact triple-mode BPF is designed based on a centrally symmetric spiral resonator. Similarly to the above-mentioned axially symmetric spiral resonator structure, two T-shaped open stubs with different lengths are also loaded on this centrally symmetric spiral resonator for three resonances generation as depicted in Fig. 10. For this filter design, we use Rogers RO4350B substrate with a relative dielectric constant of 3.48 and a thickness of $0.508 \mathrm{~mm}$. Accordingly, the design parameters in Fig. 10 are finally chosen as follows (unit: $\mathrm{mm}$ ): $L_{1}=59.3, L_{2}=4.25, L_{3}=20, L_{4}=10, L_{5}=14.2, L_{6}=19.2$, $L_{7}=3.5, \quad W_{1}=0.7, \quad W_{2}=1, \quad W_{3}=1, \quad W_{4}=1.1, \quad W_{5}=1.1$, $W_{6}=0.2, W_{7}=1.1, G=1.3, g=0.1$.

Figure 11 compares the measured results of the proposed centrally symmetric BPF with its simulations, which are in good agreement with each other. The measured cen-

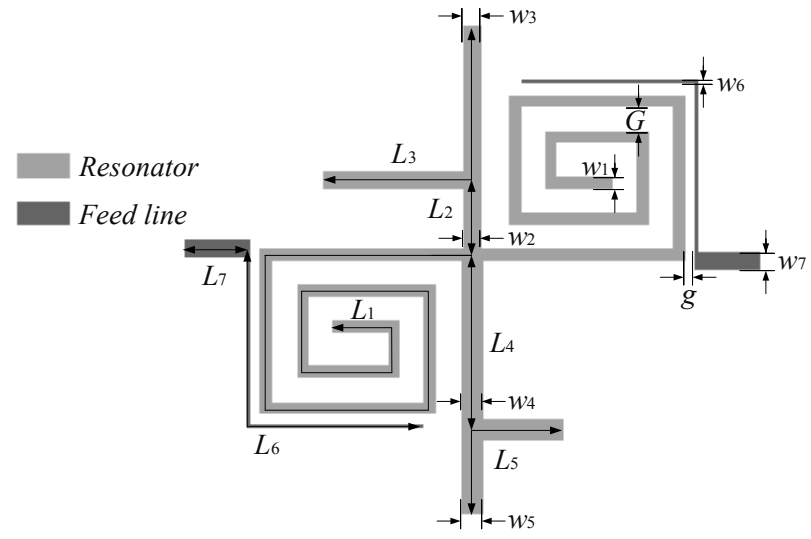

Fig. 10. Layout of the proposed centrally symmetric BPF.

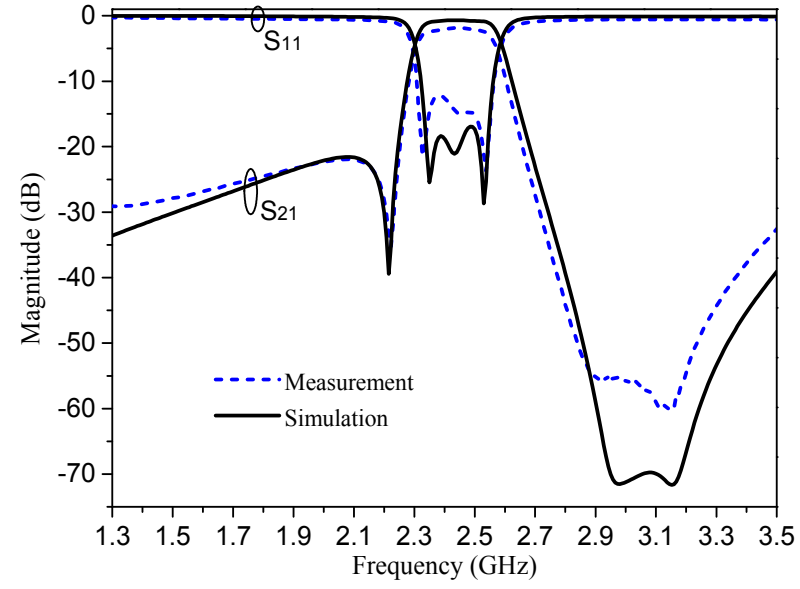

Fig. 11. Measured and simulated results of the proposed filter.

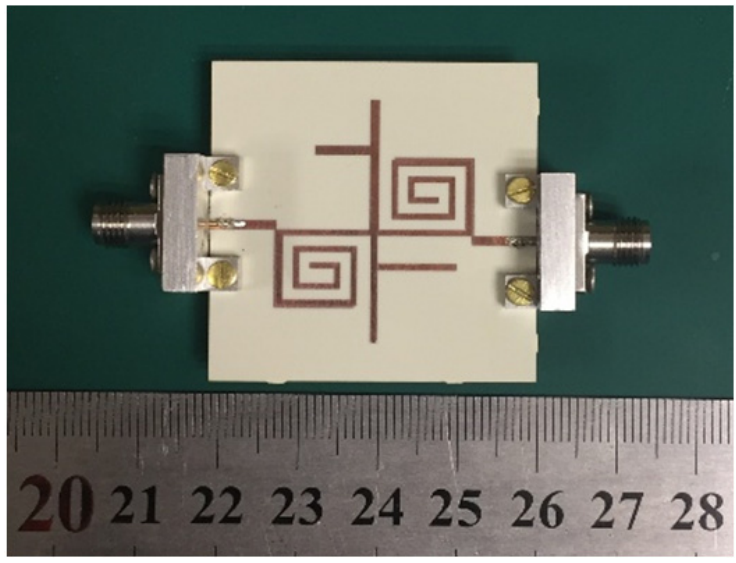

Fig. 12. Photograph of the proposed centrally symmetric BPF

ter frequency of this BPF is at $2.44 \mathrm{GHz}$ and the $3-\mathrm{dB}$ bandwidth is around from 2.32 to $2.56 \mathrm{GHz}$. The insertion losses and return losses of the measurements are less than $1.5 \mathrm{~dB}$ and over $12.2 \mathrm{~dB}$ in the passband, respectively. Figure 12 presents a photograph of the fabricated BPF using axially symmetric spiral resonator. The size of this filter is about $29.25 \mathrm{~mm} \times 27.2 \mathrm{~mm}$ corresponding to the size of $0.39 \lambda_{\mathrm{g}} \times 0.36 \lambda_{\mathrm{g}}$, where $\lambda_{\mathrm{g}}$ is the guided wavelength on the substrate at the center frequency.

\section{Conclusion}

In this paper, two compact triple-mode BPFs based on axially and centrally symmetric spiral resonators loaded with two T-shaped open stubs have been demonstrated. 
The center frequencies and bandwidths of the filters are tunable through adjustment of the resonator parameters. For the fabricated axially symmetric BPF working at $1.76 \mathrm{GHz}$, the measured insertion losses are less than $1.5 \mathrm{~dB}$ and the return losses are better than $19 \mathrm{~dB}$ in the passband. While for the fabricated centrally symmetric BPF working at $2.44 \mathrm{GHz}$, the measured insertion losses and return losses are lower than $1.5 \mathrm{~dB}$ and over $12.2 \mathrm{~dB}$ in the passband, respectively. The measured results of both fabricated filters agree with their simulations. Performance comparisons of some reported triple-mode BPFs show that the proposed axially symmetric BPF has achieved miniaturization with good frequency selectivity.

\section{Acknowledgment}

This work was supported in part by the National Natural Science Foundation of China (No. 61601390), Guangdong Natural Science Foundation (No. 2016A030310375), Natural Science Foundation of Fujian Province of China (No. 2016J05164), Young and Middleaged Teachers Education and Scientific Research Foundation of Fujian Province (No. JAT160007).

\section{References}

[1] KIM, S., KIM, N. Y. Compact bandpass filter with wide stop band response based on meandered-line stepped-impedance resonator using IPD process. Microwave and Optical Technology Letters, 2015, vol. 57, no. 6, p. 1466-1470. DOI: 10.1002/mop.29111

[2] XU, K. D., ZHANG, Y. H., LI, J. L. W., et al. Compact ultrawideband bandpass filter using quad-T-stub-loaded ring structure. Microwave and Optical Technology Letters, 2014, vol. 56, no. 9, p. 1988-1991. DOI: 10.1002/mop.28508

[3] PAN, T., SONG, K., FAN, Y. Novel wide-stopband bandpass filter with good frequency selectivity based on composite right/left handed transmission line. Microwave and Optical Technology Letters, 2012, vol. 54, no. 11, p. 2494-2497. DOI: 10.1002/mop. 27104

[4] WU, Y., HU, B., NAN, L., et al. Compact high-selectivity bandpass filter using a novel uniform coupled-line dual-mode resonator. Microwave and Optical Technology Letters, 2015, vol. 57, no. 10, p. 2355-2358. DOI: 10.1002/mop.29336

[5] ZHANG, X. Y., CHEN, J. X., XUE, Q., et al. Dual-band bandpass filters using stub-loaded resonators. IEEE Microwave and Wireless Components Letters, 2007, vol. 17, no. 8, p. 583-585. DOI: 10.1109/LMWC.2007.901768

[6] TORABI, A., FOROORAGHI, K. Miniature harmonic-suppressed microstrip bandpass filter using a triple-mode stub-loaded resonator and spur lines. IEEE Microwave and Wireless Components Letters, 2011, vol. 21, no. 5, p. 255-257. DOI: 10.1109/LMWC.2011.2122304

[7] LUGO, C., PAPAPOLYMEROU, J. Bandpass filter design using a microstrip triangular loop resonator with dual-mode operation. IEEE Microwave and Wireless Components Letters, 2005, vol. 15 , no. 7, p. 475-477. DOI: 10.1109/LMWC.2005.851573

[8] XU, K. D., ZHANG, Y. H., ZHUGE, C. L., et al. Miniaturized dual-band bandpass filter using short stub-loaded dual-mode resonators. Journal of Electromagnetic Waves and Applications,
2011, vol. 25, no. 16, p. 2264-2273. DOI: $10.1163 / 156939311798147060$

[9] DAI, G. L., ZHANG, X. Y., CHAN, C.-H., et al. An investigation of open-and short-ended resonators and their applications to bandpass filters. IEEE Transactions on Microwave Theory and Techniques, 2009, vol. 57, no. 9, p. 2203-2210. DOI: 10.1109/TMTT.2009.2027173

[10] SERRANO, L., CORRETA, F. S. A triple-mode bandpass filter using a modified circular patch resonator. Microwave and Optical Technology Letters, 2009, vol. 51, no. 1, p. 178-182. DOI: 10.1002/mop. 23950

[11] MA, X. B., JIANG, T. Compact wideband bandpass filter with controllable bandwidth and suppression of the second passband using a trimode resonator. Microwave and Optical Technology Letters, 2015, vol. 57, no. 12, p. 2939-2943. DOI: 10.1002/mop. 29475

[12] LIU, H. W., SHEN, L., JIANG, Y., et al. Triple-mode bandpass filter using defected ground waveguide. Electronics Letters, 2011, vol. 47, no. 6, p. 388-389. DOI: 10.1049/el.2011.0006

[13] MO, S. G., YU, Z. Y., ZHANG, L. Design of triple-mode bandpass filter using improved hexagonal loop resonator. Progress in Electromagnetics Research, 2009, vol. 96, no. 4, p. 117-125. DOI: 10.2528/PIER09080304

[14] XU, H., XU, K., LIU, Y., LIU, Q. H. Compact triple-mode bandpass filter using short-and open-stub loaded spiral resonator. In 2016 IEEE/ACES International Conference on Wireless Information Technology and Systems (ICWITS) and Applied Computational Electromagnetics (ACES). Honolulu (USA), 2016, 2 p. DOI: $10.1109 /$ ROPACES.2016.7465476

\section{About the Authors ...}

Kai-da XU was born in Zhejiang, China. He received the B.S. and Ph.D. degrees in Electromagnetic Field and Microwave Technology from the University of Electronic Science and Technology of China (UESTC), Chengdu, China, in 2009 and 2015, respectively. From September 2012 to August 2014, he was a Visiting Researcher in the Dept. of Electrical and Computer Engineering, Duke University, Durham, NC, under the financial support from the China Scholarship Council (CSC). He received the UESTC Outstanding Graduate Awards in 2009 and 2015, respectively. He was the recipient of the National Graduate Student Scholarship in 2012, 2013, and 2014 from the Ministry of Education, China. He is now an Assistant Professor with the Inst. of Electromagnetics and Acoustics, and Dept. of Electronic Science, Xiamen University, Xiamen, China. He has authored and coauthored over 60 papers in peerreviewed journals and conference proceedings. Since 2014, he has served as a reviewer for some journals including IEEE Transactions on Microwave Theory and Techniques, IEEE Microwave and Wireless Components Letters, IEEE Transactions on Electron Devices, IEEE Transactions on Computer-Aided Design of Integrated Circuits and Systems, IEEE Transactions on Applied Superconductivity, International Journal of RF and Microwave Computer-Aided Engineering, ACES Journal, PIER, JEMWA and so on. His research interests include $\mathrm{RF} /$ microwave and $\mathrm{mm}$-wave circuits, antennas, and nanoscale memristors. 
Meng-ze LI was born in Hunan, China. She received her B.Sc. from Hunan University, Changsha, China, in 2015, and currently she is working toward the M.S. degree in Xiamen University. Her research interests include $\mathrm{RF} /$ microwave components and circuits.

Yanhui LIU (corresponding author) was born in Guangxi, China. He received the B.S. and Ph.D. degrees both in Electrical Engineering from the University of Electronic Science and Technology of China (UESTC), Sichuan, China, in 2004 and 2009, respectively. From Sept. 2007 to June 2009, he was a Visiting Scholar in the Dept of Electrical Engineering, Duke University, Durham, NC. In July 2011, he joined the Dept. of Electronic Science, Xiamen University, Fujian, China, where he is now a Professor. He has authored and co-authored over 80 papers in peer-reviewed journals and conference proceedings. He received the UESTC Outstanding Graduate Award in 2004, and the Excellent Doctoral Dissertation Award of Sichuan Province of China in 2012. His research interests include antenna array design, array signal processing, and microwave imaging methods.

Jing AI was born in Sichuan, China. He received the B.Sc. degree in Electronic Science and Technology and M.S. degree in Electronic and Communication Engineering from the University of Electronic Science and Technology of China (UESTC), Chengdu, China, in 2007 and 2013, respectively, where he is currently working toward the Ph.D. degree in Electromagnetic Field and Microwave Technology. His recent research interests include microwave and millimeter-wave circuits and systems.

Ye-cheng BAI was born in Jiangsu, China. He received his B.Sc. from Hefei University of Technology, Hefei, China, in 2012, and currently he is working toward the M.S. degree in Xiamen University. His research interests include $\mathrm{RF} /$ microwave components and circuits. 